\title{
Chemische Ausdehnung von Sensor- und Elektrolytmaterialien bei hohen Temperaturen
}

\author{
D. Kohlmann 1 , H. Wulfmeier ${ }^{1}$, M. Schewe ${ }^{2}$, C. Rembe ${ }^{2}$, H. Fritze ${ }^{1}$ \\ ${ }^{1}$ Institut für Energieforschung und Physikalische Technologien, TU Clausthal, Goslar, Deutschland \\ 2 Institut für Elektrische Informationstechnik, TU Clausthal, Clausthal-Zellerfeld, Deutschland \\ Kontakt: dhyan.kohlmann@tu-clausthal.de
}

\section{Einleitung}

Einer der wichtigsten Versagensmechanismen von Aktivmaterialien in Sensoren, Brennstoffzellen und Akkumulatoren ist die Rissbildung bei zyklischer Beanspruchung [1], wodurch beispielsweise Brennstoffzellen durch den direkten Kontakt von Brenngas- und Luftseite zerstört werden können. Die Risse bilden sich durch mechanische Spannungen im Material, die durch Temperaturgradienten und/oder die chemische Expansion ${ }^{a}$ hervorgerufen werden [2]. Letztere entsteht bei Brennstoffzellen durch zeitlich schwankende Sauerstoffkonzentrationen und Konzentrationsgradienten zwischen der Brenngas- und Luftseite des Elektrolyten. Tritt demgegenüber eine besonders starke Expansion auf, kann der Effekt zu einer vollständig neuen Bauteilklasse führen, d. h., zu Aktuatoren, die bei hohen Temperaturen ihre Form signifikant ändern. Da Piezoelektrika mit hohen piezoelektrischen Koeffizienten durch ihre relativ niedrige Curie-Temperatur eingeschränkt nutzbar sind, besteht an dieser Stelle Bedarf an neuartigen Stellelementen z. B. zur Beeinflussung chemischer Reaktoren bei hohen Temperaturen.

Da die in den o.g. Anwendungen eingesetzten Oxide häufig als dünne Schicht ausgeführt sind [3], besteht ein Interesse, das Expansionsverhalten dünner Schichten zu verstehen. Deswegen wird hier die Expansion dünner Schichten mit Differentieller Laser-Doppler-Vibrometrie (DLDV) bei verschiedenen Sauerstoffaktivitäten untersucht.

Untersuchungsgegenstand sind dünne Schichten aus $\mathrm{CeO}_{2-\delta}, \mathrm{Ce}_{1-\mathrm{x}} \mathrm{Zr}_{\mathrm{x}} \mathrm{O}_{2-\delta}$ (CZO) und $\mathrm{Pr}_{\mathrm{x}} \mathrm{Ce}_{1-\mathrm{x}} \mathrm{O}_{2-\delta}$ (PCO). Als Substrate dienen $\mathrm{Y}_{2} \mathrm{O}_{3}$-dotierte $\mathrm{ZrO}_{2}$-Einkristalle, die als Pumpzelle für Sauerstoff fungieren. Durch Anlegen einer Spannung wird Sauerstoff transportiert, was die Sauerstoffaktivität in den abgeschiedenen Schichten verändert, sodass das Expansionsverhalten der Schichten unter reduzierenden Bedingungen untersucht werden kann. Insbesondere CZO ist aufgrund seiner Expansionseigenschaften interessant, da die Substitution von Cer durch Zirkon die Reduktionsenthalpie des Systems herabsetzt, was im Vergleich zu $\mathrm{CeO}_{2-\delta}$ zu größeren Expansionen führt [4,5]. Bei PCO lassen sich Pr-lonen bereits bei höheren Sauerstoffaktivitäten $\left(a_{\mathrm{O} 2}\right)$ reduzieren als Ce-Ionen [6]. Im Fall von PCO wird zunächst das leichter zu reduzierende Pr umgeladen, was im $a_{\mathrm{O} 2}$-Bereich oberhalb von ca. $10^{-20} \mathrm{zu}$ deutlich größeren Expansionen führt als bei $\mathrm{CeO}_{2-\delta}[7,8]$.
Die hier gemessenen Auslenkungen bei verschiedenen Temperaturen und Sauerstoffpartialdrücken werden mit Modellen verglichen. Für die Experimente werden Temperaturen $\left(T=600 \ldots 950{ }^{\circ} \mathrm{C}\right)$ und Sauerstoffaktivitäten $\left(a_{\mathrm{O} 2}=1,6 \cdot 10^{-5} \ldots a_{\mathrm{O} 2}=1,7 \cdot 10^{-24}\right)$ gewählt, bei denen die untersuchten Materialien bereits eine deutliche Expansion zeigen, jedoch noch nicht zerstört werden $[9,10]$.

\section{Stand der Forschung \\ Defektchemie und chemische Expansion}

Im Folgenden wird zur Darstellung der defektchemischen Reaktionen die Kröger-Vink-Notation verwendet [11]. In reduzierender Umgebung wird aus Metalloxiden mit vierwertigen Kationen $\mathbf{M O}_{2-\delta}$ teilweise Sauerstoff ausgebaut, wobei in vielen Fällen Sauerstoffleerstellen $\mathbf{V}_{\mathbf{0}}$ und Elektronen zurückbleiben. Die Ausbaureaktion von Sauerstoff aus dem Material lautet [3]

$$
2 \mathrm{M}_{\mathrm{M}}^{\times}+\mathrm{O}_{\mathrm{O}}^{\times} \leftrightarrow 2 \mathrm{M}_{\mathrm{M}}^{\prime}+\mathrm{V}_{\mathrm{O}}^{*}+1 / 2 \mathrm{O}_{2}(\mathrm{~g})
$$

Näherungsweise wird angenommen, dass die Konzentrationen von Sauerstoffionen $\left[\mathbf{0}_{\mathbf{0}}^{\times}\right]$und nicht-reduzierten Metallionen $\left[\mathbf{M}_{\mathbf{M}}^{\times}\right]$konstant sind. Aus dem Massenwirkungsgesetz ergibt sich damit die Gleichgewichtskonstante $\mathrm{K}$ der Reaktion (1) zu [3]

$$
\mathrm{K}=\left[\mathrm{M}_{\mathrm{M}}^{\prime}\right]^{2}\left[\mathrm{~V}_{\mathrm{O}}^{*}\right] \mathrm{p}_{\mathrm{O} 2}^{1 / 2} \text {. }
$$

Mit dem Konzentrationsverhältnis von Sauerstoffleerstellen und reduzierten Metallionen [3] $\left[\mathbf{M}_{\mathbf{M}}^{\prime}\right]=\mathbf{2}\left[\mathbf{V}_{\mathbf{0}}^{*}\right]$ folgt der Zusammenhang zwischen der Leerstellenkonzentration $\left[\mathbf{V}_{\mathbf{0}}\right]$ und dem Sauerstoffpartialdruck $\boldsymbol{p}_{\mathbf{0 2}}$ in der Umgebung:

$$
\left[\mathrm{V}_{\mathrm{O}}^{*}\right] \propto \mathrm{p}_{\mathrm{O} 2}^{-1 / 6}
$$

Die Nichtstöchiometrie $\delta$ ist mit der Leerstellenkonzentration über die Relation [3]

$$
\delta \propto 2\left[\mathrm{~V}_{\mathrm{O}}\right]
$$

verbunden. Mit Hilfe der Pumpzelle wird die Sauerstoffaktivität in den Schichten und somit auch die Nichtstöchiometrie $\delta$ eingestellt.

Das reduzierte Metallion hat häufig einen größeren Radius als das nicht reduzierte [12]. Zudem haben die entstehende Sauerstoffleerstelle eine positive sowie das reduzierte Metallion eine negative Netto-Ladung am jeweiligen Gitterplatz, was zu einer elektrostatischen Abstoßung und im Allgemeinen zu einer Vergrößerung des Abstandes der Gitterebenen bei zunehmender Reduktion führt [2,8]. Die relative Änderung des Gitterparameters $a$ in Abhängigkeit

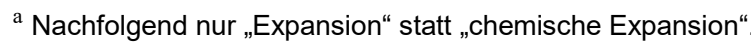




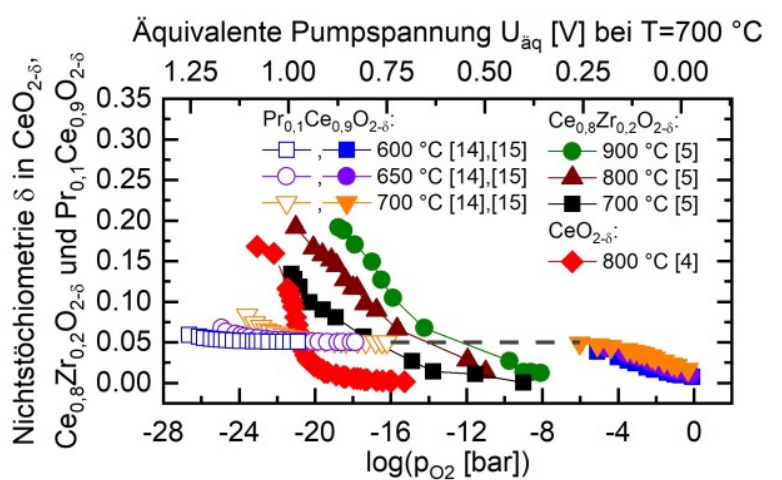

1 Abb. 1: Sauerstoff-Nichtstöchiometrie $\delta$ in den untersuchten Probensystemen $\mathrm{CeO}_{2-\delta}, \mathrm{Ce}_{0,8} \mathrm{Zr}_{0,2} \mathrm{O}_{2-\delta}$

Pro,1 $\mathrm{Ce}_{0,9} \mathrm{O}_{2-\delta}$ in Abhängigkeit des Sauerstoffpartialdrucks bei verschiedenen Temperaturen $[4,5,14,15]$.

der Nichtstöchiometrie $\delta$ wird als chemische Expansion $\varepsilon$ bezeichnet [13]:

$$
\varepsilon=\frac{\mathrm{a}-\mathrm{a}_{0}}{\mathrm{a}_{0}}=\alpha \delta \sim\left[\mathrm{V}_{\mathrm{O}}\right]
$$

Hier ist ao der Gitterparameter im stöchiometrischen Material.

In Abb. 1 ist die Nichtstöchiometrie in den untersuchten Materialien $\mathrm{CeO}_{2-\delta}$, $\mathrm{Ce}_{0,8} \mathrm{Zr}_{0,2} \mathrm{O}_{2-\delta} \mathrm{Pr}_{0,1} \mathrm{Ce}_{0,9} \mathrm{O}_{2-\delta}$ in Abhängigkeit des Sauerstoffpartialdrucks ist dargestellt. Bei PCO ist ein Plateau bei $\delta=0,05$ zu sehen, bei dem Pr vollständig dreiwertig, jedoch $\mathrm{Ce}$ noch vierwertig vorliegt. Die anderen beiden Probensysteme zeigen kein Plateau. Stattdessen steigt $\delta$ zu niedrigen Sauerstoff-Partialdrücken hin kontinuierlich an. Die Nichtstöchiometrie in $\mathrm{Ce}_{0,8} \mathrm{Zr}_{0,2} \mathrm{O}_{2-\delta}$ ist unter gleichen Bedingungen wesentlich größer als in reinem $\mathrm{CeO}_{2-\delta}$.

\section{Sphärische Verbiegung der Substrate}

Das Modell nach Stoney [14] beschreibt den Zusammenhang zwischen der lateralen mechanischen Spannung in einer dünnen Schicht und der dadurch hervorgerufenen kugelförmigen Aufwölbung des Substrats, auf dem sich die Schicht befindet.

Da bei einer festen Haftung der Schicht auf dem Substrat keine freie laterale Expansion möglich ist, entsteht in der Schicht eine der nominellen Expansion proportionale mechanische Spannung. Hierdurch wird die gesamte Probe verbogen, wie in Abb. 2 schematisch dargestellt. Die Auslenkung $D$ der Probenoberfläche ist die Summe der Schichtdickenänderung $\Delta t_{\mathrm{f}}$ und der Verbiegung $D_{0}$. Hierbe ist $\Delta t_{f}$ proportional zu $\delta$. Die Verbiegung $D_{0}$ ergibt sich nach [14] zu

$$
D_{0}=3 \varepsilon t_{\mathrm{f}} \mathrm{r}^{2} \frac{E_{\mathrm{f}}\left(1-v_{\mathrm{s}}\right)}{E_{\mathrm{s}} t_{\mathrm{s}}^{2}\left(1-v_{\mathrm{f}}\right)} .
$$

In (6) sind $\varepsilon$ die chemische Expansion in der dünnen Schicht, $t$ die Dicke, $r$ der Probenradius, E der Elastizitätsmodul und $v$ das Poisson-Verhältnis. Die Indizes $\mathrm{f}$ und $\mathrm{s}$ bezeichnen die Schicht (film) und das Substrat.

Die Schichtdickenänderung $\Delta t_{\mathrm{f}}$ ist aufgrund der weitgehenden Unterbindung lateraler Expansion um den Faktor $\left(1+v_{f}\right) /\left(1-v_{f}\right)$ gegenüber der Dickenänderung im Volumenmaterial vergrößert. Die Auslenkung $D$ berechnet sich gemäß:

$$
D=D_{0}+\Delta t_{\mathrm{f}}=\varepsilon t_{\mathrm{f}}\left(3 \mathrm{r}^{2} \frac{E_{\mathrm{f}}\left(1-v_{\mathrm{s}}\right)}{E_{\mathrm{s}} t_{\mathrm{s}}^{2}\left(1-v_{\mathrm{f}}\right)}+\frac{1+v_{\mathrm{f}}}{1-v_{\mathrm{f}}}\right)
$$

\section{Methoden und Materialien}

Probenherstellung und -aufbau

Die Schichten sind auf Substraten abgeschieden, die als Pumpzelle fungieren, bei der durch Anlegen einer Spannung Sauerstoffionen von der dünnen Schicht ins Substrat und umgekehrt gepumpt werden (Abb. 2). Die Substrate (MTI Corp., USA) bestehen aus $8 \mathrm{~mol}-\% \mathrm{Y}_{2} \mathrm{O}_{3}$ dotiertem $\mathrm{ZrO}_{2}$. Die (100)-orientierten Substrate haben eine Dicke von $320 \mu \mathrm{m}$ bis $530 \mu \mathrm{m}$ und werden zu runden Scheiben mit $2 r=10 \mathrm{~mm}$ Durchmesser gefräst (vgl. Abb. 2).

Auf die Substrate wird rückseitig eine Pt-Elektrode mittels Siebdruck und vorderseitig per gepulster Laserablation (PLD, Compex 205 pro, Lambda Physik, Deutschland) die dünne zu untersuchende Schicht aufgebracht. Die Proben werden nachträglich geglüht, um die nach der Abscheidung amorphen Schichten zu kristallisieren. Die Kristallisationstemperatur beträgt $1000^{\circ} \mathrm{C}$ für $\mathrm{CeO}_{2}$ und $900{ }^{\circ} \mathrm{C}$ für $\mathrm{Ce}_{0,8} \mathrm{Zr}_{0,2} \mathrm{O}_{2}$. Dies wird mittels Röntgen-Diffraktometrie (D5000, Siemens, Deutschland) verifiziert (Daten hier nicht dargestellt.). Anschließend wird per PLD eine PtRh-Elektrode abgeschieden. Kristalline $\mathrm{Pr}_{0,1} \mathrm{Ce}_{0,9} \mathrm{O}_{2}$-Schichten werden bei einer Temperatur von $650{ }^{\circ} \mathrm{C}$ abgeschieden. Die dünnen Oxidschichten haben einen Durchmesser von $9,5 \mathrm{~mm}$, die PLD-Elektroden von $9 \mathrm{~mm}$ und die Siebdruckelektroden von 9,5 mm. Die zu untersuchenden Schichten haben eine Dicke von $0,6 \mu \mathrm{m}$ bis $3,3 \mu \mathrm{m}$, die PLD-Elektroden von ungefähr $300 \mathrm{~nm}$. Die Dicken der Schichten und der Elektroden werden mit einem taktilen Oberflächenprofilometer (XP-2, Ambios Technology Inc., USA) an mehreren Punkten bestimmt und der Mittelwert als Schichtdicke angenommen.

\section{Elektrochemisches Pumpen}

Die Einstellung des Sauerstoffaktivität in der zu untersuchenden Schicht erfolgt durch Anlegen eines Potentials zwischen den beiden Elektroden. Die Sauerstoffaktivität $a_{\mathrm{O} 2}$ ergibt sich mit der Nernst-Gleichung [17] zu

$$
a_{\mathrm{O} 2}=p_{\mathrm{O} 2}^{\mathrm{atm}} \mathrm{e}^{-4 e_{0} U_{\text {pump }} / k_{B} T}
$$

Hierin sind $p_{\mathrm{O} 2}^{\mathrm{atm}}$ der Sauerstoff-Partialdruck der umgebenden Luft, $U_{\text {pump }}$ die Pumpspannung, $e_{0}$ die Elementarladung, $k_{B}$ die Boltzmann-Konstante und $T$ die absolute Temperatur. Damit ergibt sich beispielsweise bei $600{ }^{\circ} \mathrm{C}$ und $0,25 \vee a_{\mathrm{O} 2} \approx 10^{-7}$ und bei $600{ }^{\circ} \mathrm{C}$ und $1,00 \mathrm{~V} a_{\mathrm{O} 2} \approx 10^{-17}$. Durch Modellieren eines Spannungsteilers aus zwei in Reihe geschalteten Ohm'schen Widerständen für das Substrat und die dünne Schicht (Kontaktspannungen werden hierbei zunächst nicht berücksichtigt.) zeigt sich, dass dann die nominelle Pumpspannung als tatsächlich an der Schicht anliegendes Potential betrachtet werden kann, da der Wi- 


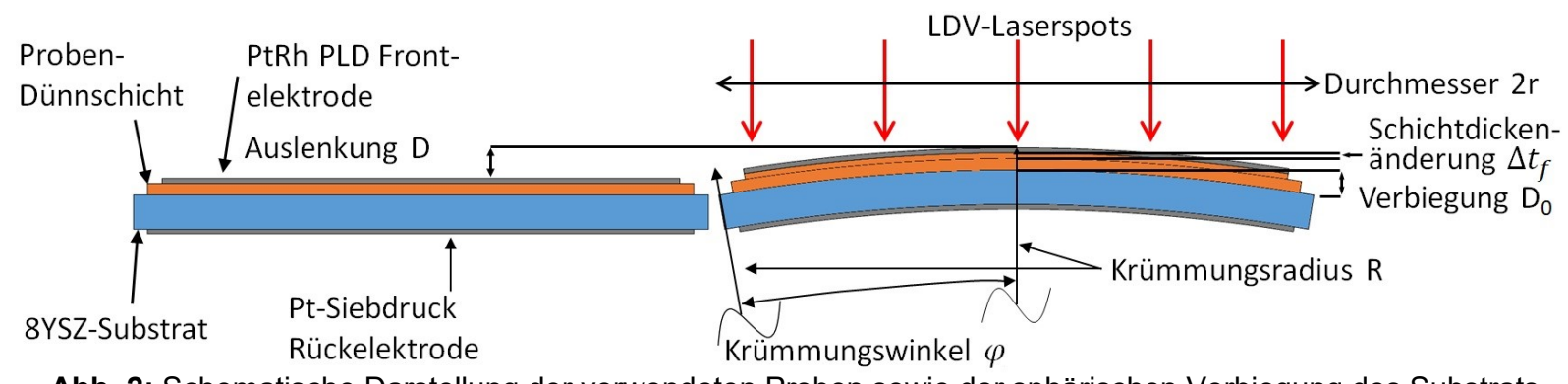

Abb. 2: Schematische Darstellung der verwendeten Proben sowie der sphärischen Verbiegung des Substrats.

derstand der Schicht sehr klein gegenüber dem des Substrats ist. Die Widerstände von Substrat und Schicht ergeben sich als Produkt aus den jeweiligen Leitfähigkeiten $[18,19]$ und der Dicke. Die Messungen der Expansion mit einem Vibrometer erfordern hinreichend schnelle Bewegungen der Oberfläche. Die Pumpspannung wird daher mit (Pump-)Frequenzen von $1 \mathrm{mHz}$ bis $10 \mathrm{~Hz}$ variiert.

Das Substrat weist einen vernachlässigbaren Expansionskoeffizienten auf [21], vgl. GI. (5), sodass die Verbiegung der Probe praktisch nur durch die Expansion der dünnen Schicht bestimmt wird.

\section{Messprinzip und -aufbau}

Es wurden zwei LDVs in Aufbauten speziell für die Hochtemperatur-Charakterisierung von Proben bis ca. $1000{ }^{\circ} \mathrm{C}$ verwendet. Diese sind ein von den Autoren realisiertes DLDV [22] und ein Einpunkt-LDV (OFV-5000, Polytec $\mathrm{GmbH}$, Deutschland), das in [25] beschrieben ist. Der DLDV-Aufbau wurde analog zum LDV-Aufbau mit vibrationsarmen, drehbar gelagerten Spiegelkippern und einem keramischen Probenhalter im Messtopf konzipiert.

Der beim Einpunkt-LDV im Messgerät verbleibende Referenzstrahl wird im Fall differentieller Messung auf den Probenrand oder -halter gelenkt. Die Laserwellenlängen betragen beim DLDV $\lambda_{\mathrm{DLDV}}=1550 \mathrm{~nm}$ und beim Einpunkt-LDV $\lambda_{\mathrm{LDV}}=633 \mathrm{~nm}$.

In Abb. 3 ist der DLDV-Aufbau schematisch gezeigt. Die hochreflektive PtRh-Elektrode und der mit $\mathrm{TiO}_{2}$ beschichtete $\mathrm{Al}_{2} \mathrm{O}_{3}$-Probenhalter reflektieren die Messstrahlen zurück ins DLDV. Dabei werden beide Strahlen auf der gesamten Weglänge möglichst eng geführt, sodass sie ähnlichen Störungen ausgesetzt sind. Bei der Interferenz von Mess- und Referenzstrahl werden der Einfluss der Längenänderung des Probenhalters durch Temperaturschwankungen und Einflüsse wie Hitzeflimmern sowie einkoppelnde mechanische Schwingungen größtenteils eliminiert. Das Pumpen der Sauerstoffionen von der dünnen Schicht ins Substrat geschieht mittels eines Funktionsgenerators (33502B, Keysight Technologies, USA) und eines spannungsgeregelten Stromverstärkers (SVM 02, MST Scientific, Deutschland). Um in der Fourier-Transformierten des Zeitsignals einen scharfen Peak zu erhalten, muss die Messdauer einem ganzzahligen Vielfachen der Anregungsperiode entsprechen. Das Zeitsignal muss vor der FourierTransformation gefiltert werden. Dies geschieht mit einem digitalen Butterworth-Bandpass 6. Ordnung mit einer Bandbreite von einer Dekade zwischen den $3 \mathrm{~dB}$-Punkten und mit der Mittenfrequenz $f_{\text {pump. }}$. Hierdurch wird die gemessene Signalamplitude bei $f_{\text {pump }}$ weniger als die Signalauflösung gedämpft.

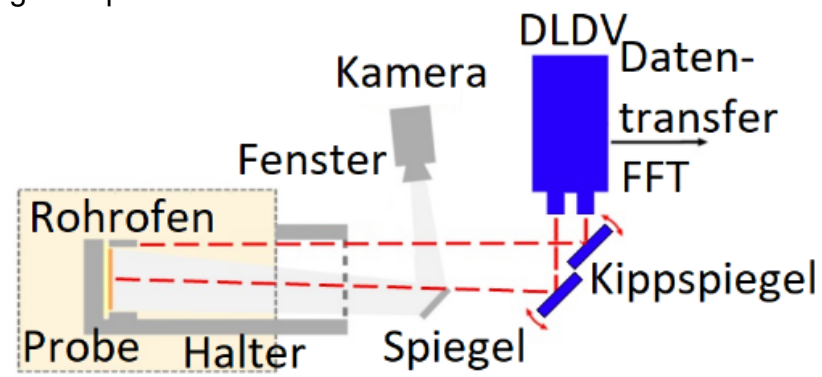

Abb. 3: Schematische Darstellung des verwendeten DLDV-Aufbaus. Mit den beiden Kippspiegeln werden die Laserstrahlen auf die Probe und deren Rand bzw. Halter gelenkt. Im Fall des Einpunkt-LDVs verbleibt der Referenzstrahl im Messgerät.

\section{Ergebnisse und Diskussion}

\section{Ceroxid}

Die Messungen an $\mathrm{CeO}_{2-\delta}$ werden mit dem Einpunkt-LDV durchgeführt und decken deswegen nur den Frequenzbereich von $0,1 \mathrm{~Hz}$ bis $10 \mathrm{~Hz}$ ab. Es wird im Temperaturbereich von $800{ }^{\circ} \mathrm{C}$ bis $950^{\circ} \mathrm{C}$ gemessen. Die Pumpspannungen betragen $0,25 \mathrm{~V}, 0,5 \mathrm{~V}, 0,75 \mathrm{~V}$ und $1 \mathrm{~V}$. Die Dicken der Schicht und des Substrats sind $t_{f}=(3300 \pm 80) \mathrm{nm}$ und $t_{s}=(327 \pm 1,6) \mu \mathrm{m}$.

In Abb. 4 sind die gemessenen Auslenkungen in der Probenmitte bei $T=900{ }^{\circ} \mathrm{C}$ gezeigt. Erwartungsgemäß nehmen die gemessenen Auslenkungen $D$ mit steigender Pumpspannung $U_{\text {pump }}$ und sinkender Pumpfrequenz $\mathrm{fzu}$. In Abb. 5 sind die bei $U_{\text {pump }}=1,0 \mathrm{~V}$ gemessenen Auslenkungen für alle vier eingestellten Temperaturen dargestellt. Es ist wieder zu sehen, dass die Auslenkungen $D$ mit abneh-

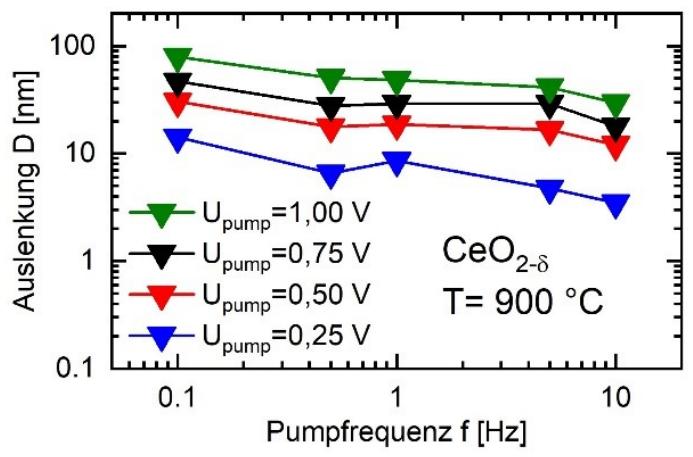

Abb. 4: Auslenkungen der $\mathrm{CeO}_{2-\delta}-$ Probe bei $T=900{ }^{\circ} \mathrm{C}$, gemessen mit dem Einpunkt-LDV. 


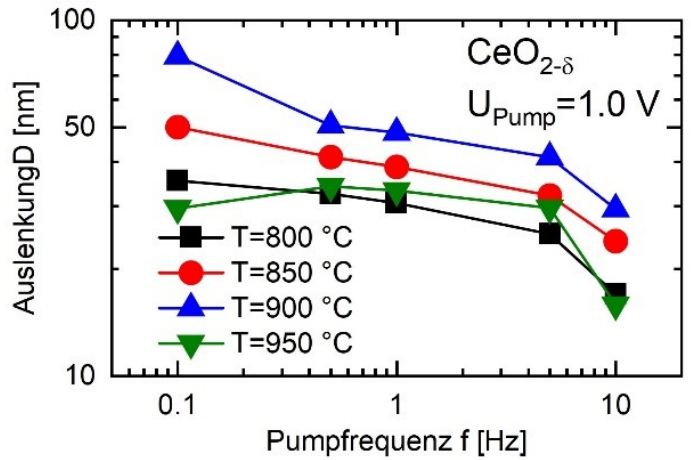

Abb. 5: Auslenkungen der $\mathrm{CeO}_{2-\delta}-$ Probe mit Pumpspannung $U_{\text {pump }}=1,0 \mathrm{~V}$, gemessen mit dem Einpunkt-LDV.

mender Pumpfrequenz $\mathrm{f}$ und bis $T=900{ }^{\circ} \mathrm{C}$ mit zunehmender Temperatur $T$ wachsen. Bei $T=950^{\circ} \mathrm{C}$ ist ein Einbruch der Auslenkung $D$ zu erkennen, der möglicherweise ein $\mathrm{Re}$ sultat der Degradation der $\mathrm{CeO}_{2}$-Schicht ist, da sich dieser Einbruch bei Nachmessungen als irreversibel herausstellte und die Elektroden noch intakt waren. Weitere Untersuchungen hierzu folgen.

\section{Cer-Zirkonium-Oxid}

An $\mathrm{Ce}_{0,8} \mathrm{Zr}_{0,2} \mathrm{O}_{2-\delta}$ werden mit beiden Vibrometern Messungen durchgeführt. Die Einpunkt-LDV-Messungen decken wieder den Frequenzbereich von $10 \mathrm{~Hz}$ bis $0,1 \mathrm{~Hz}$ ab. Die Proben werden bei $600{ }^{\circ} \mathrm{C}$ bis $800{ }^{\circ} \mathrm{C}$ unter Nutzung der Pumpspannungen $0,25 \mathrm{~V}, 0,5 \mathrm{~V}$ und $0,75 \mathrm{~V}$ charakterisiert. Die Dicken der untersuchten Schichten tf betragen ca. $1100 \mathrm{~nm}$ und die der Substrate $\mathrm{t}_{\mathrm{s}}$ ca. $325 \mu \mathrm{m}$.

In Abb. 6 sind an zwei Proben bei $T=800^{\circ} \mathrm{C}$ gemessene Auslenkungen dargestellt. Es ist wieder der Trend zu erkennen, dass die Auslenkung mit abnehmender Pumpfrequenz und zunehmender Pumpspannung $U_{\text {pump }}$ wächst. $\mathrm{Ce}_{0,8} \mathrm{Zr}_{0,2} \mathrm{O}_{2-\delta}$ zeigt deutlich größere Auslenkungen als $\mathrm{CeO}_{2}$, was sich für $U_{\text {pump }}=0,75 \mathrm{~V}$ in Abb. 7 ergibt. Bei diesem Vergleich muss beachtet werden, dass die $\mathrm{CeO}_{2-\delta}$ Probe eine dreifach dickere Schicht als die beiden $\mathrm{Ce}_{0,8} \mathrm{Zr}_{0,2} \mathrm{O}_{2-\delta}$-Proben aufweist, also bei gleicher Schichtdicke ein noch geringere Auslenkung zu verzeichnen wäre. Am Materialsystem $\mathrm{Ce}_{0,8} \mathrm{Zr}_{0,2} \mathrm{O}_{2-\delta}$ werden zusätzlich Messungen mit dem DLDV durchgeführt, da im niederfrequenten Frequenzbereich die Auflösung des Einpunkt-LDV nicht ausreicht, um die Auslenkung $D$ vom Rauschuntergrund $\Delta D$ zu trennen. In Abb. 8 ist eine Messreihe bei $T=700^{\circ} \mathrm{C}$ und

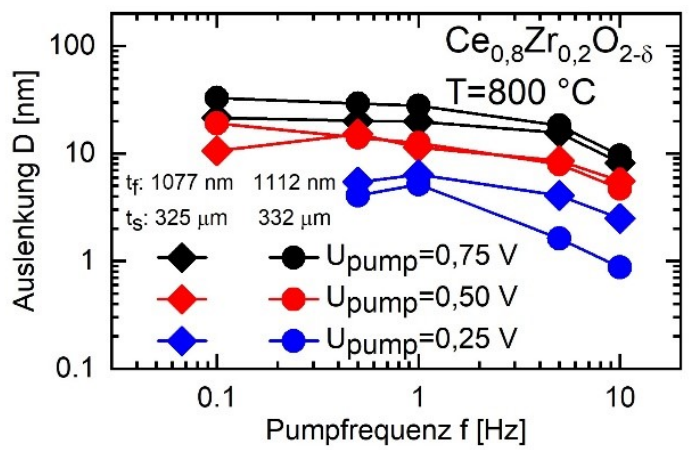

Abb. 6: Auslenkungen von zwei $\mathrm{Ce}_{0,8} \mathrm{Zr}_{0,2} \mathrm{O}_{2-\delta}$-Proben bei $T=800{ }^{\circ} \mathrm{C}$, gemessen mit dem Einpunkt-LDV.

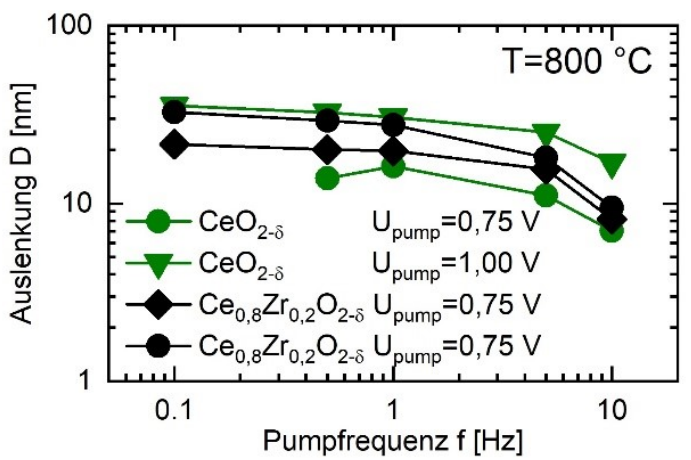

Abb. 7: Auslenkungen der $\mathrm{CeO}_{2-\delta}-\mathrm{Probe}$ und zweier $\mathrm{Ce}_{0,8} \mathrm{Zr}_{0,2} \mathrm{O}_{2-\delta}-$ Proben, gemessen mit dem Einpunkt-LDV.

$U_{\text {pump }}=0,75 \mathrm{~V}$ gezeigt. Als Rauschmittelwert wurde der Effektivwert aus \pm 20 Kanälen um $f_{\text {pump }}$ in der Fourier-Transformierten genommen. Dabei entspricht die Auflösungsbandbreite $\Delta f$ der Messung der inversen Messdauer. Sie beträgt für die dargestellten Messungen $11,5 \mu \mathrm{Hz}$, $22,9 \mu \mathrm{Hz}, 45,8 \mu \mathrm{Hz}, 91,7 \mu \mathrm{Hz}, 183,4 \mu \mathrm{Hz}, 518,3 \mu \mathrm{Hz}$, $1,04 \mathrm{mHz}, 4,15 \mathrm{mHz}$ und $7,95 \mathrm{mHz}$, in der Ordnung aufsteigender Frequenz. Datenpunkte, die das Rauschniveau $\Delta D$ um weniger als den Faktor 2 übertreffen $(\mathrm{SNR}<3 \mathrm{~dB})$ sind nicht dargestellt. Es ist ein Abflachen sowohl der Auslenkung als auch des Rauschens zu niedrigen Frequenzen hin zu beobachten.

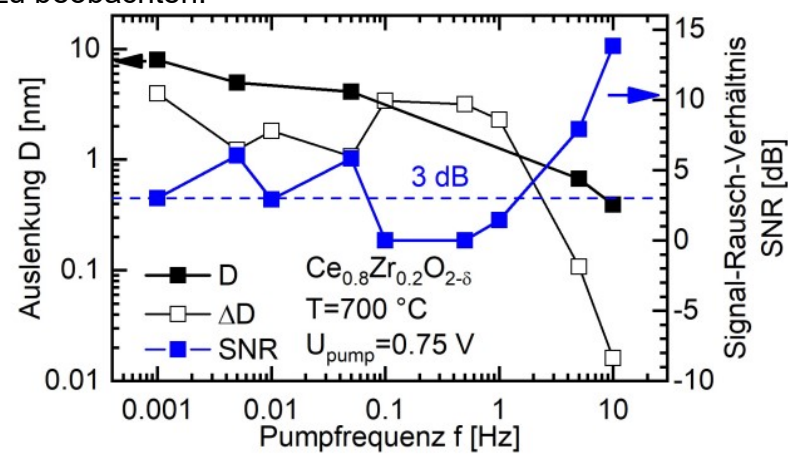

Abb. 8: Auslenkung einer $\mathrm{Ce}_{0,8} \mathrm{Zr}_{0,2} \mathrm{O}_{2-\delta}$-Probe bei $T=$ $700{ }^{\circ} \mathrm{C}$, gemessen mit dem DLDV.

\section{Praseodym-Cer-Oxid}

An $\operatorname{Pr}_{0,1} \mathrm{Ce}_{0,9} \mathrm{O}_{2-\delta}$ wurden mit beiden LDVs Messungen durchgeführt. In Abb. 9 sind an derselben Probe gemes sene Daten dargestellt. Gemessen wird hier die Verbiegung

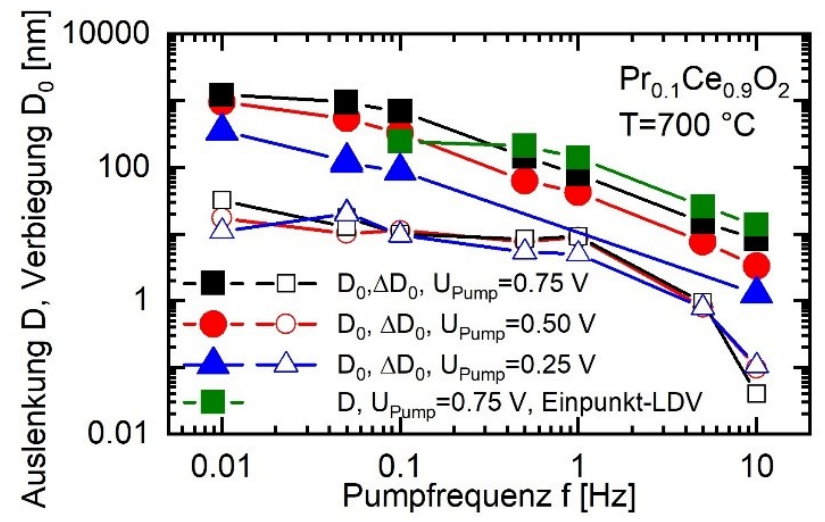

Abb. 9: Verbiegung einer $\mathrm{Pr}_{0,1} \mathrm{Ce}_{0,9} \mathrm{O}_{2-\delta}$-Probe bei $T=$ $700^{\circ} \mathrm{C}$, aufgenommen mit dem Einpunkt-LDV und dem DLDV. 
der Probe ohne die Schichtdickenänderung, da die Laserstrahlen des DLDV auf dem Probenrand und in der Probenmitte positioniert waren. Die Substratdicke beträgt $t_{s}=520 \mu \mathrm{m}$, die Schichtdicke $t_{f}=1350 \mathrm{~nm}$. Die mit beiden Vibrometern gemessenen Werte stimmen gut überein, was sich anhand der Daten für $U_{\text {pump }}=0,75 \mathrm{~V}$ ergibt. Da die Messkurven mit sinkender Frequenz abflachen und zumindest bei $U_{\text {pump }}=0,75 \mathrm{~V}$ für $f<0,1 \mathrm{~Hz}$ näherungsweise waagerecht verlaufen, kann davon ausgegangen werden, dass bei $f=0,01 \mathrm{~Hz}$ der stationäre Zustand erreicht ist.

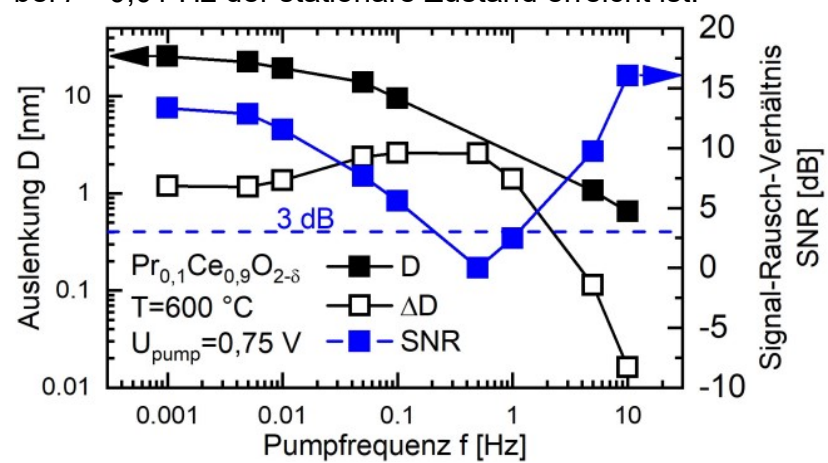

Abb. 10: Auslenkungen einer $\operatorname{Pr}_{0,1} \mathrm{Ce}_{0,9} \mathrm{O}_{2-\delta}$-Probe bei $T=600^{\circ} \mathrm{C}$, gemessen mit dem DLDV.

In Abb. 10 sind an einer weiteren $\operatorname{Pr}_{0,1} \mathrm{Ce}_{0,9} \mathrm{O}_{2-\delta}$-Pobe mit einer Schichtdicke von $\mathrm{t}_{\mathrm{f}}=590 \mathrm{~nm}$ und einer Substratdicke von $\mathrm{t}_{\mathrm{s}}=525 \mu \mathrm{m}$ bei $T=600{ }^{\circ} \mathrm{C}$ aufgenommene Auslenkungen gezeigt. Das Abflachen der Auslenkungen im niedrigen Frequenzbereich ist auch hier zu sehen. Ein Maximum des Rauschens ist bei $f_{\text {pump }}=0,1 \mathrm{~Hz}$ bis $1 \mathrm{~Hz}$ zu verzeichnen. Bei der niedrigsten Pumpfrequenz von $f_{\text {pump }}=0,001 \mathrm{~Hz}$ beträgt das Rauschen $\Delta D=1,2 \mathrm{~nm}$ bei einer Auflösungsbandbreite von $\Delta f=11,5 \mu \mathrm{Hz}$.

\section{Diskussion}

Da keine Literaturdaten zu den Elastizitätsmoduln und Poisson-Verhältnissen von PCO und CZO für die vorliegenden Bedingungen zur Verfügung stehen, werden hier stattdessen ersatzweise diejenigen von $\mathrm{CeO}_{2}$ verwendet [23] Die aus diesen Literaturdaten und eingestellten Pumpspannungen nach (7) berechnete Auslenkung der $\mathrm{CeO}_{2-\delta-P r o b e}$ bei $T=800{ }^{\circ} \mathrm{C}$ und $U_{\text {pump }}=1,0 \mathrm{~V}$ beträgt $D_{800 \text {,theo }}^{\mathrm{CeO}-\delta}=3000$ $\mathrm{nm}$. Die gemessene maximale Auslenkung beträgt $D_{800, \text { mess }}^{\mathrm{CeO} 2-\boldsymbol{\delta}}=32,5 \mathrm{~nm}$ und verfehlt den erwarteten Wert um den Faktor 92. Die für $T=900{ }^{\circ} \mathrm{C}$ und $U_{\text {pump }}=1 \mathrm{~V}$ berechnete Auslenkung beträgt $D_{900 \text {,theo }}^{\mathrm{CeO}-\delta}=12600 \mathrm{~nm}$, die gemessene Auslenkung von $D_{900 \text {, mess }}^{\mathrm{CeO}}=79,4 \mathrm{~nm}$ bleibt um einen Faktor von 158 hinter der Erwartung zurück.

Die an den CZO-Proben gemessenen Auslenkungen sind ebenfalls deutlich kleiner als erwartet. Der aus [23] nach (7) berechnete Wert beträgt $D_{800, \text { theo }}^{\text {CZO }}=730 \mathrm{~nm}$ bzW. $739 \mathrm{~nm}$ und wird um den Faktor 23 bzw. 34 verfehlt. Die mit (7) aus [14,15, 23,24] für die in Abb. 9 gezeigten Messungen an PCO berechnete Verbiegung beträgt $D_{700 \text {,theo }}^{P C O}=1200 \mathrm{~nm}$. Der Messwert von $1219 \mathrm{~nm}$ stimmt hiermit gut überein. Die für die in Abb. 10 gezeigten Messungen berechnete Auslenkung beträgt $D_{600 \text {,theo }}^{P C O}=546 \mathrm{~nm}$, der Messwert von 26 $\mathrm{nm}$ verfehlt dies um den Faktor 21.
Die Gründe für die Diskrepanz zwischen berechneten und gemessenen Auslenkungen müssen erforscht werden. Aufnahmen mit einem Rasterelektronenmikroskop (hier nicht gezeigt) zeigen Risse in den dünnen Schichten, die der Verbiegung des Substrates entgegenstehen. Eine Abschätzung der Ausdehnung beim Aufheizen anhand der mittleren Rissweite sowie des Abstands der Risse ergab jedoch, dass diese sich aufgrund der Differenz der thermischen Ausdehnungskoeffizienten der Substrate und der dünnen Schichten [21] beim Aufheizen auf $T=800^{\circ} \mathrm{C}$ wieder schließen müssten. Es ist denkbar, dass sich die Risse nicht komplett schließen und die Schichten auch Poren aufweisen. Die isotherme Expansion des Materials würde in diesem Fall nicht zu einer Verbiegung führen, sondern zum Verschließen der Poren.

\section{Schlussfolgerungen}

Es konnte gezeigt werden, dass die Mischsysteme $\mathrm{Ce}_{0,8} \mathrm{Zr}_{0,2} \mathrm{O}_{2-\delta}$ und $\mathrm{Pr}_{0,1} \mathrm{Ce}_{0,9} \mathrm{O}_{2-\delta}$ eine größere Expansion zeigen als reines $\mathrm{CeO}_{2-\delta}$. Bei $\mathrm{Pr}_{0,1} \mathrm{Ce}_{0,9} \mathrm{O}_{2-\delta}$ wird das erwartete Verhalten beobachtet, dass sich die Auslenkungen für die verschiedenen gewählten Pumpspannungen zu niedrigen Frequenzen hin annähern. $\mathrm{CeO}_{2-\delta}$ und $\mathrm{Ce}_{0,8} \mathrm{Zr}_{0,2} \mathrm{O}_{2-\delta}$ hingegen zeigen kein solches Verhalten. Die Kurven der Auslenkungen bei den verschiedenen Pumpspannungen liegen weit auseinander. $\mathrm{CeO}_{2-\delta}$ degradiert offensichtlich erst bei Temperaturen über $900{ }^{\circ} \mathrm{C}$ und zeigt eine höhere Temperaturbeständigkeit als die beiden anderen Probensysteme, die bereits bei deutlich niedrigeren Temperaturen von $800{ }^{\circ} \mathrm{C}$ absinkende Auslenkungen zeigen.

Obwohl $\mathrm{Ce}_{0,8} \mathrm{Zr}_{0,2} \mathrm{O}_{2-\delta}$ unter gleichen Bedingungen größere Nichtstöchiometrien $\delta$ zeigt als reines $\mathrm{CeO}_{2-\delta}$, übertreffen die an $\mathrm{Ce}_{0,8} \mathrm{Zr}_{0,2} \mathrm{O}_{2-\delta}$-Proben gemessenen Auslenkungen diejenigen an $\mathrm{CeO}_{2-\delta}$-Proben nicht im selben Maß. Diese relativ geringe Ausdehnung der hier vorliegenden $\mathrm{Ce}_{0,8} \mathrm{Zr}_{0,2} \mathrm{O}_{2-\delta}$-Schichten legt daher nahe, dass sie z. B. für die Anwendung als Sauerstoffreservoir in Katalysatoren besser geeignet als $\mathrm{CeO}_{2-\delta}-\mathrm{Schichten}$ sind.

Die hohen Auslenkungen der $\operatorname{Pr}_{0,1} \mathrm{Ce}_{0,9} \mathrm{O}_{2-\delta}$-Proben sowie deren geringe Abhängigkeit von der Pumpspannung demonstrieren die Eignung des Materials für Aktuatoren.

Weiterhin wird gezeigt, dass mit dem DLDV in einstelligen $\mathrm{mHz}$-Bereich gemessen werden kann. Die ermittelten Auslenkungen stimmen mit denen des Einpunkt-LDV überein.

Die Auflösung des Einpunkt-LDV beträgt bei der niedrigsten Messfrequenz von $0,02 \mathrm{~Hz}$ und Raumtemperatur ca. $40 \mathrm{~nm}$ [25]. Bei $720^{\circ} \mathrm{C}$ und $1 \mathrm{~Hz}$ ergibt sich ein Wert von $18 \mathrm{~nm}$. In dieser Arbeit werden bei $700{ }^{\circ} \mathrm{C}$ und $0,001 \mathrm{~Hz}$ mit dem DLDV Auflösungen von $6 \mathrm{~nm}$ bei einer Auflösungsbandbreite von $11,5 \mu \mathrm{Hz}$ erreicht (Abb. 10). Das verbesserte System ermöglicht somit die Detektion deutlich geringerer Auslenkungen bei gleichzeitig höherer Messgenauigkeit. Dies bestätigt den Ansatz, zur Unterdrückung von Störeffekten bei hohen Temperaturen differentiell zu messen. Im Frequenzbereich von $0,1 \mathrm{~Hz}$ bis $1 \mathrm{~Hz}$ liegt ein starkes Rauschen vor, das nicht gänzlich eliminiert werden konnte (s. Abb.8-10). Es wird vermutet, dass dieser Beitrag von 
menschlichen Einflüssen herrührt. Störungen durch $\mathrm{Ge}$ bäudeschwingungen können jedoch nicht ausgeschlossen werden.

\section{Literatur}

[1] ATKINSON, A.: Solid Oxide Fuel Cell Electrolytes-Factors Influencing Lifetime. Academic Press (2017)

[2] BISHOP, S.R. ; MARROCCHELLI, D. ; CHATZICHRISTODOULOU, C. ; PERRY, N.H. ; MOGENSEN, M.B ; TULLER, H.L. ; WACHSMAN, E.D.: Chemical Expansion: Implications for Electrochemical Energy Storage and Conversion Devices. In: Annual Review of Materials Research Vol. 44 (2014)

[3] PREUX, N. ; ROLLE, A. ; VANNIER, R.N.: Electrolytes and ion conductors for solid oxide fuel cells (SOFCs). In: Functional Materials for Sustainable Energy Applications, Woodhead Publishing, S. 370-401 (2012)

[4] BISHOP, S.R. ; DUNCAN, K.L. ; WACHSMAN, E.D.: Defect equilibria and chemical expansion in non-stoichiometric undoped and gadolinium-doped cerium oxide. In: Electrochimica Acta, Vol. 54, S. 1436-1443 (2009)

[5] BISHOP, S. R. ; NAKAMURA T. ; AMEZAWA, K.: Chemically-induced expansion of $\mathrm{Zr}_{0.2} \mathrm{Ce}_{0.8} \mathrm{O}_{2-\delta}$. In: Solid State lonics Vol. 261, S. 1-4 (2014)

[6] WULFMEIER, H. ; KOHLMANN, D. ; DEFFERRIERE, T. ; STEINER, C. ; MOOS, R. ; TULLER, H.L. ; FRITZE, $H$.: Thin-film chemical expansion of ceria based solid solutions: laser vibrometry study. In: Z. Phys. Chem. (2021)

[7] BISHOP, S.R. ; STEFANIK, T., ; TULLER, H.L.: Defects and transport in PrxCe1-xO2- $\delta$ : Composition trends. In: Journal of Materials Research, Vol. 27, S.2009-2016 (2012).

[8] SCHMITT, R. ; NENNING, A. ; KRAYNIS, O. ; KOROBKO, R. ; FRENKEL, A. I. ; LUBOMIRSKY I. ; HAILE, S. M. ; RUPP, J. L. M.: A review of defect structure and chemistry in ceria and its solid solutions. In: Chem. Soc. Rev. Vol. 49, S. 554-592 (2020)

[9] JEN, H.W. ; GRAHAM, G.W. ; CHUN, W ; MCCABE, R.W. ; CUIF, J.P. ; DEUTSCH, S.E. ; TOURET, O.: Characterization of model automotive exhaust catalysts: Pd on ceria and ceria-zirconia supports. In: Catalysis Today, Vol. 50, Iss. 2, S. 309-328 (1999)

[10] BORCHERT, H. ; FROLOVA, Y.V. ; KAICHEV, V.V ; PROSVIRIN, I.P. ; ALIKINA, G.M. ; LUKASHEVICH, A.I. ; ZAIKOVSKII, V.I. ; MOROZ, E.M. ; TRUKHAN, S.N. ; IVANOV, V.P. ; PAUKSHTIS, E.A. ; BUKHTIYAROV, V.I. ; SADYKOV, V.A.: Electronic and Chemical Properties of Nanostructured Cerium Dioxide Doped with Praseodymium. In: J. Phys. Chem. B Vol. 109, S. 5728-5738 (2005)

[11] KRÖGER, F.J. ; Vink, A.H.: Relations between the concentrations of imperfections in crystalline solids. Solid State Physics Vol. 3, S.307-435, 1956

[12] SHANNON, R. D.: Revised Effective Ionic Radii and Systematic Studies of Interatomie Distances in Halides and Chaleogenides. In: Acta Cryst. Vol. 32, S. 751-767 (1976)

[13] MARROCCHELLI, D. ; BISHOP, S.R. ; TULLER, H.L.; YILDIZ, B.: Understanding Chemical Expansion in NonStoichiometric Oxides: Ceria and Zirconia Case Studies. In: Adv. Funct. Mater., Vol. 22, S. 1958-1965 (2012)

[14] CHEN, D. ; BISHOP, S.R. ; TULLER, H.L.: Nonstoichiometry in Oxide Thin Films Operating under Anodic
Conditions: A Chemical Capacitance Study of the Praseodymium-Cerium Oxide System. In: Chem. Mater. Vol. 26 Iss. 22, S. 6622-6627 (2014)

[15] CHEN, D. ; BISHOP, S. R. ; TULLER, H. L.: Non-stoichiometry in Oxide Thin Films: A Chemical Capacitance Study of the Praseodymium-Cerium Oxide System. Adv. Funct. Mater. Vol. 23, S. 2168- 2174 (2013)

[16] JANSSEN, G.C.A.M. ; ABDALLA, M.M. ; VAN KEULEN, F. ; PUJADA, B.R. ; VAN VENROOY, B.: Celebrating the 100th anniversary of the Stoney equation for film stress: Developments from polycrystalline steel strips to single crystal silicon wafers. In: Thin Solid Films Vol. 517, Issue 6, S.1858-1867 (2009)

[17] FEINER, A.S. ; MCEVOY, A.J.: The Nernst Equation. In: Journal of Chemical Education, Vol. 71, No. 6 (1994)

[18] IKEDA, S. ; SAKURAI, O. ; UEMATSU, K. ; MIZUTANI, N. ; KATO, M.: Electrical conductivity of yttria-stabilized zirconia single crystals. In: Journal of Materials Science Vol. 20, S. 4593-4600 (1985)

[19] KOGUT, I. ; STEINER, C. ; WULFMEIER, H. ; WOLLBRINK, A. ; HAGEN, G. ; MOOS, R. ; FRITZE, H.: Comparison of the electrical conductivity of bulk and film $\mathrm{Ce}_{1-\mathrm{x}} \mathrm{Zr}_{\mathrm{x}} \mathrm{O}_{2-\delta}$ in oxygen-depleted atmospheres at high temperatures. In: J. Mater. Sci., Vol. 56, S. 1719117204 (2021)

[20] WELLER, M. ; HERZOG, R. ; KILO, M. ; BORCHARDT, G. ; WEBER, S. ; SCHERRER, S.: Oxygen mobility in yttria-doped zirconia studied by internal friction, electrical conductivity and tracer diffusion experiments. In: Solid State lonics, Volume 175 (2004), Issues 1-4, S. 409-413,

[21] BISHOP, S. R. ; DUNCAN, K. L. ; WACHSMAN, E. D.: Thermo-chemical expansion of SOFC materials. In: ECS Transactions, Vol. 1 Iss. 7, S. 13-21 (2006)

[22] SCHEWE, M. ; KOHLMANN, D. ; WULFMEIER, H. ; FRITZE, H. ; REMBE, C.: Methoden zur Minimierung des Rauscheinflusses durch Hitzeflimmern bei einem heterodynen Laser-Doppler-Vibrometer. In: tm - Technisches Messen. Vol. 87, Issue s1, pp. 44-49, Aug. 2020.

[23] WACHTEL, E. ; LUBOMIRSKY, I.: The elastic modulus of pure and doped ceria. In: Scripta Materialia Vol. 65 , S. 112-117 (2011)

[24] BISHOP, S.R. ; TULLER, H.L. ; KURU, Y. ; YILDIZ, B.: Chemical expansion of nonstoichiometric Pro.1 $\mathrm{Ce}_{0.9} \mathrm{O}_{2-\delta}$ : correlation with defect equilibrium model. In: J. Eur. Ceram. Soc. Vol. 31, S. 2351-2356 (2011)

[25] SCHMIDTCHEN, S. ; FRITZE, H. ; BISHOP, S. ; CHEN, D. ; TULLER, H.L.: Chemical expansion of praseodymium-cerium oxide films at high temperatures by laser doppler vibrometry. In: Solid State lonics, Vol. 319 (2018), S. 61-67

\section{Danksagung}

Die Autoren danken der Deutschen Forschungsgemeinschaft (Förderkennzeichen FR1301/31-1 und RE3980/3-1), sowie dem Forschungszentrum Energie-Speichertechnologien. Weiterer Dank gilt der Arbeitsgruppe um Prof. Harry L. Tuller (MIT) für die Abscheidung der PCO-Schichten sowie der Arbeitsgruppe um Prof. Ralf Moos (Universität Bayreuth) für die Herstellung der $\mathrm{CZO}$ - und $\mathrm{CeO}_{2}$-Targets für die PLD-Abscheidung. 